\title{
Very Unusual Presentation of Pancreatic Cancer Revealed by Traumatic Spleen Injury
}

\author{
Kwang Yeol Paik \\ Department of Surgery, The Catholic University, Yeouido St. Mary’s Hospital, Seoul, South Korea. \\ Email:szpaik@gmail.com
}

Received August $13^{\text {th }}, 2012$; revised September $16^{\text {th }}$, 2012; accepted September $28^{\text {th }}, 2012$

\begin{abstract}
Accidental diagnosis of pancreatic ductal adenocarcinoma (PDAC) follow by trauma is very rare. Splenic infarction is unusual presentation of PDAC, and splenic rupture associated with PDAC has been reported in few cases so far. We present a patient with PDAC revealed by splenic laceration after blunt abdominal trauma. The 64-year-old patient visited emergency center with left flank pain derived from slipping down on the floor. The patient was diagnosed with splenic rupture, and was intended to perform urgent splenectomy. During operation, distal pancreas was resected with spleen due to possible severe chronic pancreatitis. However, he was diagnosed as having pancreas tail cancer with remnant cancer on the resected margin. We performed radiacal antegrade modular pancreatectomy (RAMPS) seven days after initial operation. We present a case of pancreatic tail adenocarcinoma revealed by blunt abdominal trauma which we recognized accidentally after trauma. Detail evaluation were performed on this patient according to history, previous imaging studies which lead to possible early diagnosis.
\end{abstract}

Keywords: Pancreatic Ductal Adenocarcinoma; Splenic Injury

\section{Introduction}

Early diagnosis of pancreatic ductal adenocarcinoma (PDAC) is very difficult even if patient presented with some symptoms. Splenic infarction or splenic rupture are exceedingly unusual presentation of PDAC [1-5]. In pancreas head cancer, painless jaundice, upper abdominal pain may be clinical presentation. If the cancer is located in the body or tail, weight loss and back pain may be the first symptoms to develop. Pancreas tail cancer can invade the vascular structure in splenic hilum, subsequently, splenic infarction and rupture or pseudoaneurysm of splenic artery can occur. Since the diagnosis of PDAC only with the clinical symptoms is difficult, radiologic evaluation such as computed tomography (CT) scan, magnetic resonance imaging (MRI) is needed. So far, there has not been any reported patient with PDAC by trauma. Herein, we present a patient with PDAC revealed by spleen laceration due to blunt abdominal trauma.

\section{Case Presentation}

A 64-year-old male patient visited emergency room complaining of left flank pain after slipping down on the floor. He had no history of alcoholics and any other disease in abdomen. His abdomen was rigid, rebound tenderness was checked on the whole abdomen. He showed stable vital sign (heart rate 80/min, blood pressure 110/60 $\mathrm{mmHg}$ ). The hemoglobin ( $\mathrm{Hb})$ was $12.3 \mathrm{~g} / \mathrm{dL}$, platelet count was 251,000 $\mathrm{mm}^{3}$. Abdominal CT scan revealed hemoperitoneum with spleen laceration Grade II [6] and calcification of pancreas tail suggestive of chronic pancreatitis (Figure 1). After checking CT, he showed low blood pressure $80 / 60 \mathrm{mmHg}$, Hb level decreased to 8.5 $\mathrm{g} / \mathrm{dL}$. We decided to undergo emergency laparotomy owing to rapid hemoglobin change and severe abdominal pain. During surgery, his peritoneal cavity was filled with about $1000 \mathrm{~mL}$ of blood. The spleen was decapsulated and half of surface was lacerated with active hemorrhage. Pancreas tail was hard and was abutted the mesocolon and omentum. Nonetheless we intended the splenectomy although splenic hilum could not finely identified owing to severe atrophied pancreas which was abutted the hilum. Short gastric vessels were engorged suggestive of splenic vessel thrombosis or stenosis. In our case, traumatic splenic injury might induced hemoperitoneum. Transformation of vessel or pancreas related to PDAC did not induced bleeding. We performed, distal pancreatectomy with splenectomy. Pancreas tail portion showed yellowish firm fibrotic change (Figure 2). The pancreas transaction performed by blade and the stump of pancreas was ligated by non-absorbable suture. We did not have any doubt about the pancreatic malignancy. He was getting better without any complication. On 6th 

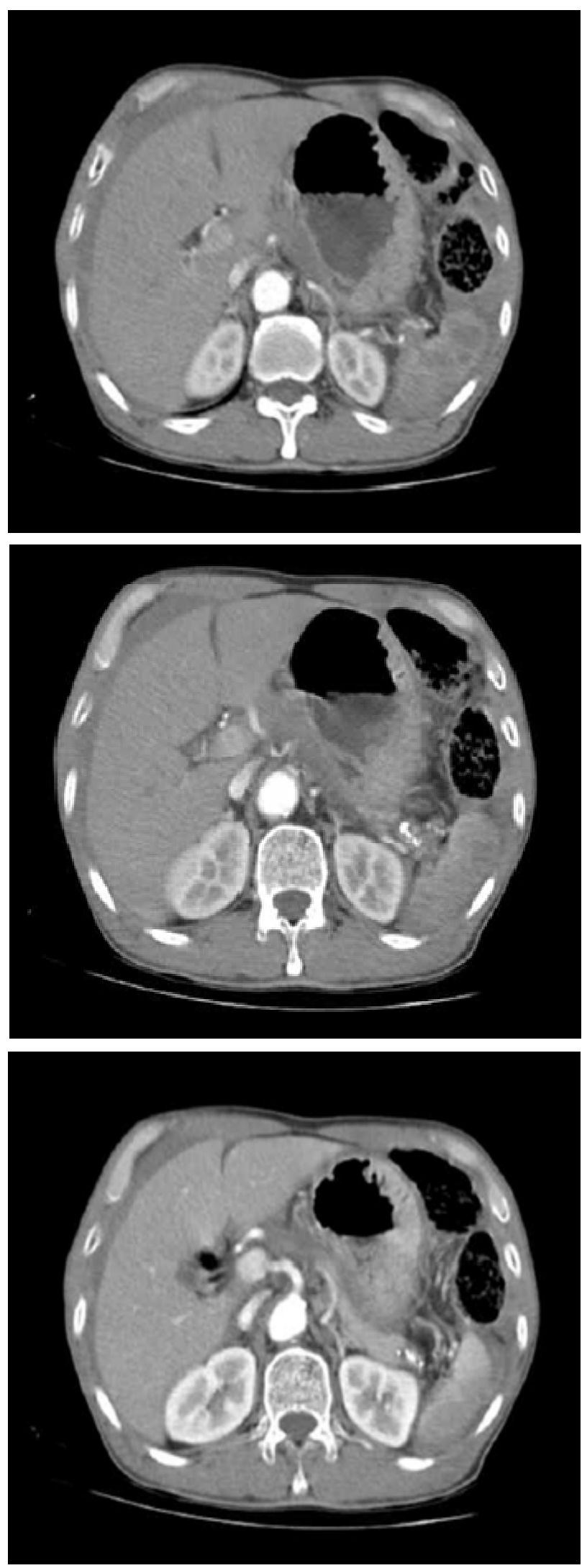

Figure 1. Upper abdominal CT scan revealed hemoperitoneum with splenic laceration.
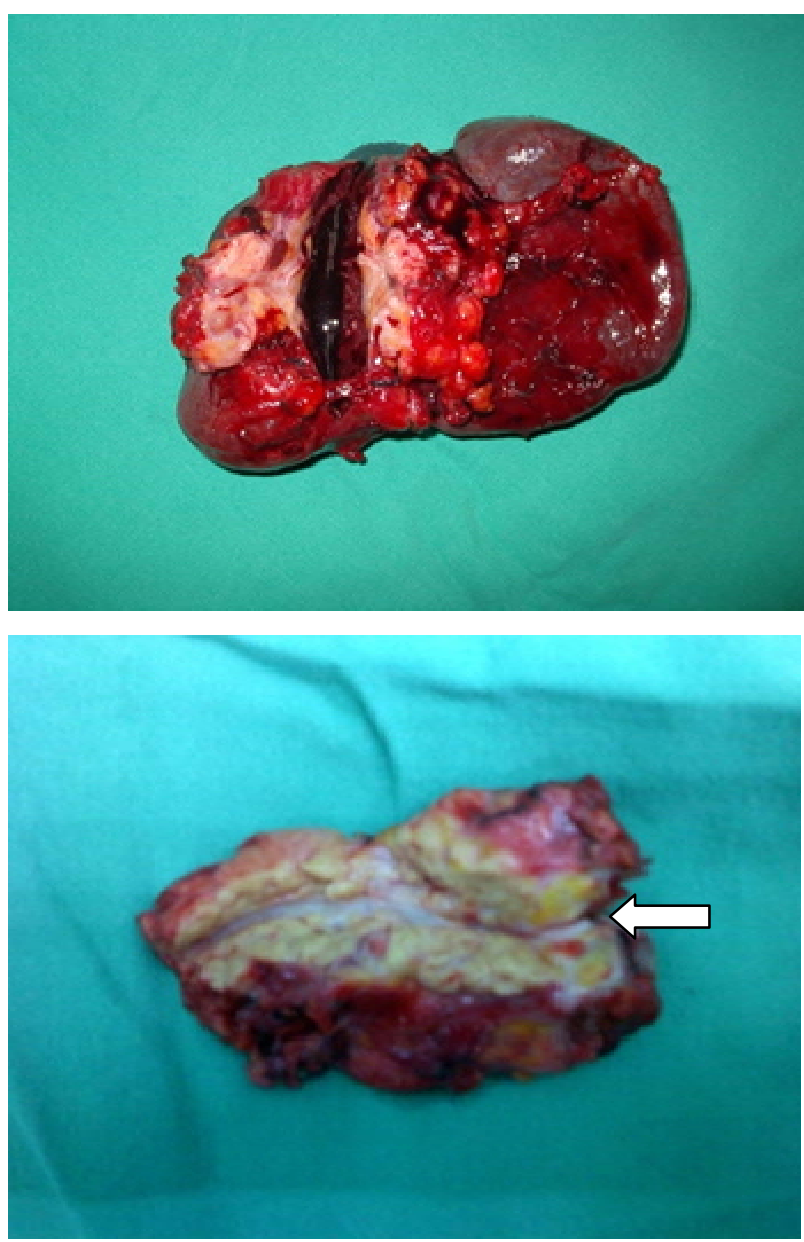

Figure 2. The gross specimen of spleen and distal pancreas, PDAC do not invade the splenic hilum. Resection margin of pancreas show tumor infiltration by pathology. White arrow indicate the distal margin of additional resected pancreas.

days after surgery, pathologist reported the pancreatic ductal adenocarcinoma and the residual tumor was detected on resection margin. We decided on additional operation, and performed modified radical antegrade pancreatectomy (RAMPS) at 7th days after initial surgery (Figure 3). Our institutional policy for body to tail pancreas cancer treatment is RAMPS since 2007. We performed pancreatectomy at the level of splenoportal junction. Peripancreatic lymph nodes were not enlarged, and para-aortic nodes were negative by frozen biopsy. Permanent pathologic reported T2N0 (0/38) M0 according to 6th TNM stage with well differentiation. The total tumor size was $25 \mathrm{~mm}$ confined to the pancreas, and tumor size on additional resected specimen was $5 \mathrm{~mm}$. There was no pancreatic fistula and small bowel ileus occurred at 7 days after second surgery, and was discharged 28th days after 2nd surgery. In spite of our institutional experience with adjuvant chemotherapy for T2 pancreas cancer, we did not perform any adjuvant treat- 


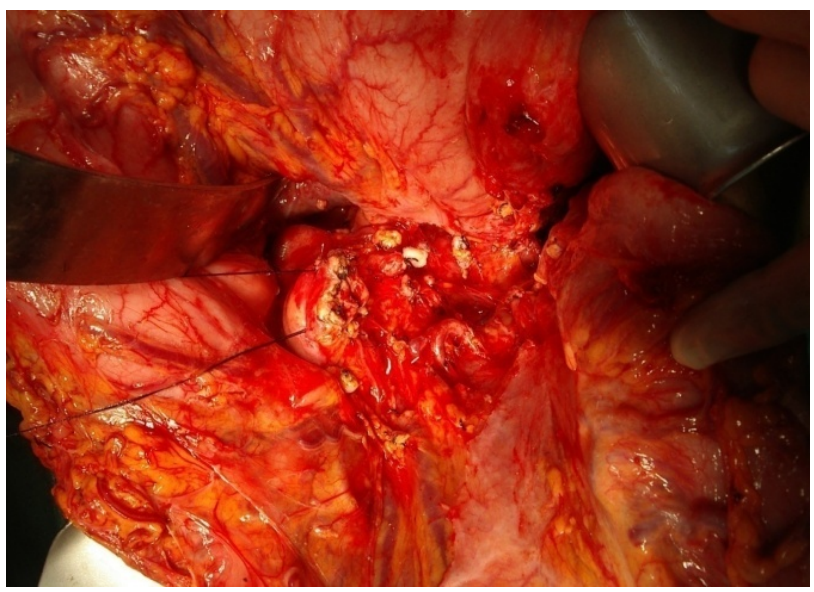

Figure 3. Operative field that radical antegrade modified pancreatectomy was performed.

ment for him due to refusal of therapy. Currently despite his refusal, he lived for three years after surgery without any recurrence or metastasis.

\section{Discussion}

Splenic rupture is extremely rare sign of pancreatic cancer, that only six cases were reported. Previous reported six cases of rupture did not occurred due to trauma, but instead rupture of splenic artery aneurysm, and direct invasion of PDAC. Pancreas tail cancer can invades splenic hilum and also inside the splenic parenchyma, causing splenic ischemia and vein occlusion, that this may represent the most important factor in the process of splenic rupture. The present patient is first case of pancreatic cancer presenting as traumatic splenic laceration. The patient was diagnosed as pancreas cancer by trauma ironically, he is considered lucky due to relatively early detection of cancer.

Pancreas cancer is associated with vague symptoms, especially, body to tail cancer rarely develops any symptoms. In imaging study such as CT, the differential diagnosis between chronic pancreatitis and PDAC sometimes difficult, with or without mass formation. CT scan has been used in an attempt to distinguish chronic pancreatictis from pancreas cancer in many studies. However it has been investigated with limited success [7]. There has been some report that splenic rupture is associated with PDAC [1-5]. So far, only six cases were reported, splenic rupture was derived from splenic ischemia with cancer invasion to splenic vessels. However, traumatic rupture of spleen with PDAC diagnosis was first in our case. Unusual pattern of PDAC presentation were pseudocyst, aneurysm of splenic vessel [8,9]. These findings did not always exclude the possibility of pancreatic cancer [9].
The patient is living three years after surgery without any recurrence or metastasis. We performed adjuvant therapy after pancreatectomy beyond T2N0, however, the patient refused the chemotherapy. We present a case of pancreatic tail adenocarcinoma revealed by blunt abdominal trauma. First one to recognized pancreatic cancer by trauma accidentally, that prudent suggestion and evaluation was performed on this patient according to history and previous imaging study, there by leading to early diagnosis.

\section{REFERENCES}

[1] S. Chung, K. Park and A. K. Li, "A Pancreatic Tumor Presenting as a Ruptured Spleen," HPB Surgery, Vol. 1, No. 2, 1989, pp. 161-163. doi:10.1155/1989/82783

[2] V. Patrinou, G. Skroubis, V. Zolota and C. Vagianos, "Unusual Presentation of Pancreatic Mucinous Cystadenocarcinoma by Spontaneous Splenic Rupture,” Digestive Surgery, Vol. 17, No. 6, 2000, pp. 645-647. doi:10.1159/000051979

[3] E. Yettimis, V. Trompetas, N. Varsamidakis, N. Courcoutsakis, V. Polymeropoulos and E. Kalokarinos, "Pathologic Splenic Rupture : An Unusual Presentation of Pancreatic Cancer," Pancreas, Vol. 27, No. 3, 2000, pp. 273-274. doi:10.1097/00006676-200310000-00015

[4] W. M. Smith, J. G. Lucas and F W. L. rankel, "Splenic Rupture-A Rare Presentation of Pancreatic Carcinoma," Archives of Pathology and Laboratory Medicine, Vol. 128, No. 10, 2004, pp. 1146-1150.

[5] M. Wong, J. Y. See, W. Sufyan and R. K. Diddapur, "Splenic Infarction. A Rare Presentation of Anaplastic Pancreatic Carcinoma and a Review of the Literature," Journal of Pancreas, Vol. 9, No. 4, 2008, pp. 493-498.

[6] E. E. Moore, C T. H. ogbill, G. J. Jurkovich, S. R. Shackford, M. A. Malangoni and H. R. Champion, "Organ Injury Scaling: Spleen and Liver (1994 Revision),” Journal of Trauma, Vol. 38, No. 3, 1995, pp. 323-324. doi:10.1097/00005373-199503000-00001

[7] E. M. Remer, M. E. Baker, "Imaging of Chronic Pancreatitis,” Radiologic Clinics of North America, Vol. 40, No. 6, 2002, pp. 1229-1242. doi:10.1016/S0033-8389(02)00044-1

[8] K. Y. Paik, S. H. Choi, J. S. Heo and D. W. Choi, "Solid Tumors of the Pancreas Can Put on a Mask through Cystic Change," World Journal of Surgical Oncology, Vol. 19, No. 9, 2011, p. 79. doi:10.1186/1477-7819-9-79

[9] H. Furukawa, N. Fukushima and K. Shimada, "Splenic Artery Aneurysm Secondary to Pancreatic Carcinoma," American Journal of Gastroenterology, Vol. 95, No. 12, 2000, pp. 3659-3660. doi:10.1111/j.1572-0241.2000.03401.x 